\title{
Programa de formación en servicios bibliotecarios y de información para la atención a las comunidades indígenas rurales mexicanas Año 2002
}

\author{
MARÍA DEl Rocío Gra NiElPARRA \\ CentroUniversitariodeInvestigacionesBibliotecológicas \\ delaUNAM, 04510, Méxi co D.F., Tel: 56- 23- 03- 29. \\ E-mail:rgraniel@ servidor.unam.mx \\ Martha AliCia AÑoRVE GUILlÉN \\ CentroUniversitariodeInvestigacionesBibliotecológicas \\ delaUNAM, 04510, Méxi co D .F., Tel: 56- 23- 03- 29. \\ E-mail:anorve@ servidor.unam.mx \\ Filiberto Felipe Martínez Arellano \\ CentroUniversitariodeInvestigacionesBibliotecológicas \\ delaUNAM, 04510, Méxi co D .F., Tel: 56- 23- 03- 29. \\ E-mail:felmar@ servidor.unam.mx
}

\begin{abstract}
RESUMEN
Elpresentedo cumento derivadelain vestigación co lectivainterdiscipli nariaeinterinstitu cio naldeno minada"Pro gramadeformaciónderecur sosbiblio tecariosbilin gües", ypresen taelpro gramadeformación téc ni caparalosbiblio tecariosqueaten deránaco munidadesindígenasrurales, sustentado en una descripción general de estasco munidades, supro ble mática de servicios bibliotecarios, sus necesidades de información y las características básicas de tales servicios para estas comunidades. ${ }^{1}$
\end{abstract}
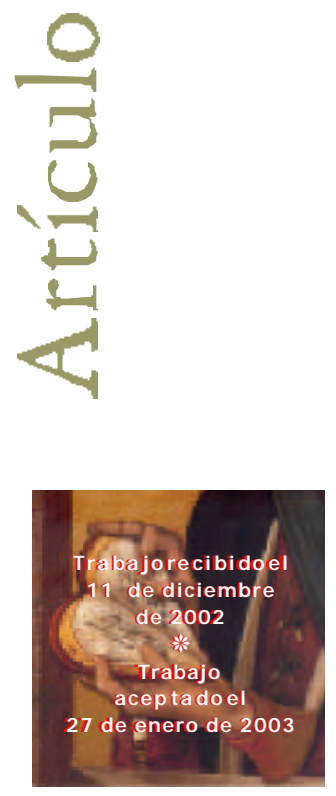

Palabrasclave: For ma ción técnicabiblioteca ria, Educación bibliotecaria, For mación de bibliotecariosindígenas.

1 Eldo cumen to baseparaestepro gramafueelabo rado porlasinvestigado rasdelCen tro Universitario deInvestigacionesBiblio tecoló gicas(CUIB) MarthaAliciaAñorveGuillényMaríadel Ro cío G raniel Parra, quieneslo so metieronalarevisión tan to delosacadémicosdeedu caciónin dígena delaUniversidad Pedagó gicaNacio nalydel Cen tro deEstudiosparaelD esarrollo Rural,Pro des, Aso ciación Civil, como delosbiblio tecariosdelaD irección $G$ eneraldeEducación In dígenaydel Instituto Nacio nalIndigenista,ytambién como delossiguien tesinvestigado resdel CUIB: Lina Escalo naRíosy Filiberto FelipeMartínezArellano.Ésteúl timo participó conlasautorasoriginales del do cumen to demaneraconstan tehastasuversión final. 


\title{
FORMATION PROGRAM ON LI BRARYANDINFOR MATIONSER VI CES INTENDED TO ATTEND INDIANRURAL MEXICAN COMMUNITIES MARÍA DEL ROCÍO GRANIEL-PARRA \\ MARTHA ALICIA AÑ ORVE-GUILLÉN \\ Filiberto Felipe MARTínEZ-ARELlano
}

\begin{abstract}
ABST RACT
Thispaperderivesfromacollectiveinterdisciplinarianandinter-institutional research called "Pro gram formation forbilin guallibrarian resources" and de velopsthepro gram of technical formation for theli brarians who will attend rural indian communities. It is a general description of these communities, the problems they face regarding librarian services, their information needs and thebasiccharacteristics theseservicesneed to com ply with in order to fit their needs.

Key Words: Te chni cal li brary, Li brary Edu ca tion, In dian li brary edu ca tion,.
\end{abstract}

\section{PRE SENT ACIÓn GE NERAL}

$\mathbf{H}$ astaelmo mento sepuedeafirmarqueen laRepúblicaMexicanasólo espo rádi camenteexistenserviciosbiblio tecariosquesepro pongan respon derrealmentealascaracterísticaslingüísticas, culturalesyru ralesdelasco munidadesindígenas. ${ }^{2}$ En un país que avanza políticamente hacia la democracia resulta necesario hacer efectivo el derecho de ese sector de la sociedad a estar informado.

La bibliotecología considera que es fundamental preparar los recursos humanos no únicamen teparaaten derlademan daactual, sino tam bién parapo derenfrentarel desarrollobiblio tecario quepo dríaimpulsarseenelpaís.

Preo cupadosporaten derlosserviciosbiblio tecariosdirigidosalasco munidades indígenas que se sustenten en su diferenciación cultural y lingüística, ${ }^{3}$ en 1998 el CUIB empren diólainvestigaciónso bre "Lainformaciónenlascomunidadesin díge nasapartirdelosserviciosbiblio tecariosen México", delacual sederivó un proyecto específico de carácter colectivo denominado "Programa de formación de recursos

$2 \mathrm{Al}$ averiguar la ubicación de servicios bibliotecarios para las comunidades indígenas mexicanas quecon tem plen su diferen ciacul turaly ylin gǘstica, seen con tró queel Municipio de Zautla, en la SierraNortedelEstado dePuebla, pro porcio naestosserviciosbiblio tecarioslaorganizaciónno gubernamentaldenominada Cen tro deEstu diosparael D esarrollo Rural (CESDER). Tales servi ciosfueron el pun to departidaquenosllevó aplan tearlanecesidad deformarperso nalbiblio teca rio para las comunidades indígenas. En algunas comunidades de dicho Municipio funcionan biblio tecasesco lares, académicasypúblicasqueatien den alapo blación ruralin dígena con un enfoque bilingüe-intercultural. Es, pues, en el ám bi to no gu bernamen tal don desehan empezado a implementar servicios bibliotecarios con ese enfoque.

3 Laspoblacionesindígenastienenpredo minantementetendenciascolectivasyoralesen laco muni cación, en ellas do minalalen gua maternaso brelalen guafran cadel país que es el es pañol, y cuentan con un alto grado deanal fabetismo realyfun cio nal. 
bibliotecariosbilingües" cuyo objetivo esprecisamentediseñaropcioneseducativas paraformarpersonalquediseñe, dirijayoperetalesservicios.

Elestudio queaportó loselementosbásicos ${ }^{4}$ paradiseñarlasop cioneseducativas fueel realizado enlasbiblio tecasyaexistentesen lasco munidadesruralesnahuatdel Estado dePuebla, quefueronim pulsadasporlaorganizaciónno gubernamentaldenominadaCentrodeEstudiosparaelD esarrollo Rural( CESDER).

En un primermo mento el proyecto co lectivo seplan teabaexclusivamentelanecesidad dedesarro llarel plan deestudio so breformación delicen ciadosen biblio tecolo gíaparaaten dercomunidadesindígenas. No obstantelarealidaddelperso nalbiblio tecario queoperalosserviciosbiblio tecariosdeCESD ER ydeaquellasinstituciones gubernamentales y no gubernamentales que están tratando de impulsar tales servicios, plan teó lanecesidad dediseñarno sólo lo an teriorsino ademásun pro gra ma de formación decarác ter eminen temen tetécnico, elcual sedesarro lla en el pre sentedocumento.

\section{DESCRIPCIÓN GENERAL DE LAS COMUNIDADES IN DÍGENASRURALES}

Las comunidades indígenas de México padecen serias carencias socioeconómicas, po líticaseinformativas. ElInstituto Nacio naldeEstadísticaG eo grafíaeI nformática (INEGI), registró en el censo de 1990 que el 7.9\% de la población total de nuestro paísesin dígena, porcentajequerepresenta6,411,952millo nesdehabitantes conestascaracterísticas.PorsuparteelInstituto NacionalIndigenista( INI)consideró que el $10.7 \%$ de la po blación denues tro países in dígena, lo quenos dalacifrade 8,709,688dein dígenas. ${ }^{5}$

Respecto de los gruposétnicosqueconstituyenlapo blaciónin dígenamexicana, el cen so delINEG I de 1990 reconoce la exis ten ciadese sen taycuatro gru posylenguasétnicos. Estosdatospermiten un primeracercamien to aladiversidad culturaly lin güísticain dígena, yeviden cian lanecesi dad detrabajartenien do en cuentaesadiversidadquegenéricamenteguardaciertosrasgosco munesdeterminadostanto por lacondiciónindígenacomoporlacondiciónrural.

Ladiversidadculturalylingüísticamexicanapresentarasgosco munesquesemani fiestan enaspectostalescomo,sucosmo visiónin dígenaysusformasdein terpretarlas relacio nesconlanaturaleza, susespaciospro ductivos, lamaneradeen ten deryexplicar elmun doy, so bretodo, suformaparticulardeen ten dersusrelacio nesinternasyhacia la sociedad mayoritaria. La tierra es el centro de su vida y el medio de supervivencia

4 Maríadel Ro cío G raniel Parra. LascommidadesindígenasylosserviaosbibliotecariosenMéxicaunestudio decasa MéxiœLaA u to ra, 2002. (Tesis- Maes traen Biblio teco lo gía-UNAM, FacultaddeFilo so fía y Letras, Maestríaen Biblio teco lo gía).367p.

5 Arnul fo Em briz, Luis Arce, «al. IndicadaressociceconómicosdelospuedosindígenasdeMéxiç México, D.F. InstitutoNacional Indigenista, Dirección deinvestigaciónypro mo ción cultural, Subdirección de investigación, 1993, p. 23-25. 
prin cipalal quesearraigan no obstan teel es tado crítico en queésteseen cuen tra, que losim pulsaalamigración tem po ral o permanen tecomo com plemento o sustitu ción desusobrevivenciaeconómica. ${ }^{6}$

Los resultados de la política educativa mexicana en las comunidades indígenas sonalarman tesyremitenaaltosgradosdeanal fabetismo realyfun cio nal demodo tal que el $43 \%$ de la población indígena mayor de 15 años no tiene ningún tipo de instrucción y es analfabeta, cerca del 31\% tiene primaria incompleta, el 14\% primaria completa y solamente el $12 \%$ estu diosmásalládelaprimaria. ${ }^{7} \mathrm{D}$ e esta formaen los pueblosin diosprevalecelaoralidad, lo cual significaqueno puedenhacerefectivo el ejerciciodelosderechosalainformaciónescritayalalectura.

A pesardequeel perfil delasco munidadesnahuatdelaSierraN ortedePueblano esto talmentegeneralizable, sí permitióidentificar algunasdelascaracterísticas pre valecientesentrelascomunidadesin dígenas,talescomo: 8

su identidad étnica como representación de su diferencia respecto de la sociedad mayoritaria,

* un dominio oral de la lengua materna,

* el uso oral y elemental, por exigencia, de la len guafran caen un alto por centaje de la población indígena,

* el excepcional porcentaje de alfabetas en su lengua materna,

* un alto porcentaje de analfabetismo funcional en la lengua materna,

* mayores porcentajes de analfabetas en español,

* mayores porcentajes de analfabetismo funcional en la lengua franca,

* bajos grados y niveles de escolaridad, preponderantemente en la lengua franca, y de calidad deficiente.

D e varios estudios antropológicos, ${ }^{9}$ lingüísticos ${ }^{10}$ y educativos indígenas, ${ }^{11}$ derivamoslossiguientesrasgosgenéricos:

* variación de alcances en la normalización escrita de las lenguas indígenas,

* alto grado de estigmatizaciónde su propia cul tu raylen gua(rechazo u ocul tamiento de ellas),

6 Guillermo BonfilBatalla.Méxicoprouundaunaaivilizadớnecada México, Edito rial G rijal bo,1989, p. 53-72.

7 Embriz, pait.

8 Maríadel Ro cío G raniel Parra.LascommidadesindígenesylosserviacosbibiotecariosenMéxicaunestudio decasa Méxica Op cit, p. 187-190.

9 Sesu gierenal gunasreferen ciasbásicas:deG uillermo BonfilBatallaPensamuestraaltbra y México profundaunaaivilizadónnegada; deAlfredo Ló pez A ustinCurpohumamoeideølogáallasconcepoionesdelos antiguos nahuas y de Leif Korsbaek Introdurciónal sistemadecangos antoloǵa

10 MaríadelRo cío GranielParra,."Aspectoslingüísticosaconsiderarenbibliotecasdirigidasalascomunidades indígenas mexicanas". MétodbsdeInformacón Sep tiem bre, 1999.

11 Sesu gierenal gunasreferen ciasbásicas:PdíticasingǘsticasenMéxicaCom pilado porBeatrizG arza Cuarón.México:UNAM, Centro de Investigaciones interdisciplinariasen Cien ciasyHumanida des, 1997, 270 p. Co lec ción:Lademo craciaen México. ElbadeG uz mán G o mez.V ocesindígenas eelucaaónbilingiedialtural enMéxico México:Direc ción G eneral dePublicacio nesdelConsejo $\mathrm{Na}$ cio nal paralaCul turaylas Artes, Institu to Nacio nal In digenista, 1991,159p. 
* relación asimétrica con la sociedad mayoritaria,

* bajo reconocimiento de los indígenas como ciudadanos del país.

Las formas y medios informativosque pudieran ser comunes a otras comunidadesruralessonlassiguientes: 12

* mayoruso deformasco lec tivasen labúsqueda, transmisiónyob ten ción de la información,

* mayor socialización grupal de la información,

* mayor uso de la oralidad en el flujo de la información,

* uso obligado de la información por medio de la lectoescritura, ya sea por medio de terceros o directamente,

* poco uso de la información escrita.

Elcúmulo delascaracterísticasan tesmen cio nadasdebeserconsiderado al diseñarlosserviciosbiblio tecariosydeinformación paralasco munidadesindígenas, los cualesnopuedenseridénticosalosdelaso ciedadmayo ritaria.

\section{NECESIDADES DE INFORMACIÓN DEL USUARIOIN DÍGENA}

Toda vez que los serviciosbiblio tecariosdeben respon deralasatisfacción delas necesidades de información de las comunidades a las que van dirigidas, la presente pro puestadeformacióntécnicasesustentaenlascaracterísticasylaidentificación de lasnecesidadesdein formación delasco munidadesindígenas.Porotraparteelsondeo permanente de éstas es objeto de los propios contenidos formativos de dicha propuesta.

Al igual que otras comunidades las de los indígenas tienennecesidadesdeinformaciónyéstasestán relacio nadasconsuentorno, su co tidianidadyelmejo ramiento desunivel devida. Unain formaciónidó neaen lo an teriorpo dríaayudarlesamejo rar sus condiciones económicas, políticas, sociales educativas, culturales y fortalecería suidentidad.

Parasatisfacerestasnecesidadesdeinformaciónesimportanteidentificaralosindígenascomo usuariosdeserviciosbiblio tecariosyto maren cuentasuscaracterísticasétnicas,educativasydecomunicación.

Talescaracterísticassehan men cio nado como rasgosgenéricosdelasco munida desin dígenasru ralesdenuestro país, guardan relación con elen torno cam pesino na cional y remiten a comunidades alejadas de la com pren sión delo escrito.Portan to estasnecesidadesdeinformación secen traneninformacióndecarácterru ral quees obtenidafundamentalmenteporlavíaoralycolectiva.

Enelcitado estu dio delasco munidadesin dígenasdelEstado dePueblaseiden ti ficó unperfildenecesidadesdeinformación quecubrelastemáticasquesein dican a

12 Maríadel Ro cío G raniel Parra. LascommidadesindígenesylosserviaosbibiotecariosenMéxicaunestudio decasa Méxica Op cit, p. 189. 
continuaciónyquerepresentanunacercamientogenérico alasnecesidadesdeinformaciónquetienenlascomunidadesindígenasruralesdenuestro país. ${ }^{13}$

* Alimentación

* Atención de la salud

* Bienestar social

* Comercialización

- Costumbres y tradiciones indígenas, religiones y otras culturas

* Apoyo a los niveles educativos

* Gestión y tramitación de servicios públicos

* Legislación y derechos humanos y ciudadanos

* Acontecimientos políticos y de gobierno

* Producción diversificada campesina y fuentes de ingreso

* Elaboración del vestido

Vivienda

Si bien la mayoría de los rubros anteriores son temáticas informativas de interés paralaso ciedadmexicanaensuconjunto, enlasco munidadesin dígenaséstasco bran maticesparticularesrelacionadosconlapo brezadesuentornocampesino.

Lasparticularidadesdeinformaciónquepudieran circunscribirseen estosrubros dependen delascaracterísticasdecadazonain dígenaylaespecificidad desupro pio con tex to, queim plicael tipo derecursosespecíficoscon losquecuen tanylaforma en la que éstosseapro vechan. Unaspecto genérico queprevaleceenlasco munida des indígenas y filtra las características de sus necesidades de información es que constituyenlossectoresconmayorescaren ciasso cioeconómicasdelpaís.

\section{SERVICIOS BIBLIOTECARIOS PARA COMU NID ADESIN DÍGENAS}

Existen ya servicios bibliotecariospara las comunidadesindígenas, en trelos que seencuen tran losdelaD irección G eneraldeBiblio tecas(D GB) deCo nacultaylosde algunas organizacio nesno gubernamen tales. Sedestacael caso delosserviciosproporcionadosporelCentro deEstudiosparaelD esarrolloRural(CESDER).

\section{Dirección G eneral de Bibliotecas ${ }^{14}$}

La D GB instala bibliotecas públicas en cabeceras municipales y localidades con más de mil habitantes.Peroúnicamente,2,303co munidadesindígenas ${ }^{15}$ reúnen tal requisito;sinembargo no to dasellascuentanconbiblio tecaspúblicas.

13 Maríadel Ro cío G raniel Parra. LascommidadesindígenasylosserviaiosbibiotecariosenMéxicaunestudio decasa Méxica Opat, p. 191-203.

14 Información obtenidaen elcurso "Biblio tecaspúblicasen México",im partido porlaD irección General de Bibliotecas, Conaculta,México, D.F. Del 17 al 19 de junio de 1997.

15 Arnulfo Embriz,eal. qp at, p. 29-31. 
Además se puede afirmar que las colecciones y servicios bibliotecarios que se le ofrecenaesebajo porcentajedeco munidadesin dígenasrurales, no respon denasus necesidadesdeinformación, pueslaco lecciónbásicaconlaquecuen tatodabiblio tecapúblicarespondemásbienalasnecesidadesdeinformacióndelaspo blacionesurbanas.Tam po co sein cluyenmaterialesenlalen guain dígenamaternaqueprevalece en esasco munidades. Sin embargo aunqueestascoleccio nesestán formadasprinci palmente por materiales impresos, también incluyen materiales auditivos, audiovisualeso gráficos, queesossíseríannecesarios paralasco munidadesin dígenas, dada sutendenciaalacomunicaciónoral-visual.

También sucedequelosserviciosbiblio tecariosno satisfacenlasnecesidadesdelas co munidadesin dígenasporqueéstasrequieren queseincluyaprincipalmenteunserviciodeconsultaquerespondaalavíaoral predo minantementeenlen guamaternaydela manerapreferentementecolectivaquecaracterizaaestasco munidades. Talesservicios no con tem planlasdiferen ciasculturalesnilin güísticas, ademásdequelosrecursoshumanosquelosbrin dan no sonlosadecuados, porqueno manejan lalen guain dígenay desconocen el contexto de esas comunidades y los servicios bibliotecariosque deberíanserlesofrecido.

\section{Organismos no gubernamentales ${ }^{16}$}

Varias organizaciones no gubernamentales en los estados de Chiapas y Puebla realizan esfuerzos por establecer bibliotecas en comunidades indígenas que tomen en cuentasu diferen ciaculturalylingüística. En el primercaso seen cuen tran sietebibliotecas de la zona zapatista de Chiapas que se han propuesto operar servicios biblio tecariosparaestasco munidades, perolasituación deguerrano hapermitido que estosserviciosoperen conregularidad.Elsegundo caso eseldelCen tro deEstu dios parael D esarrollo Rural(CESD ER), que hastaelmo men to haestablecido nuevebi bliotecas que sí pretenden proporcionar servicios que respondan adecuadamente a las diferencias culturales, lingüísticas y al contexto propio de dichas comunidades. Asípues, dado queexisten en nuestro país 18,341 co munidadesin dígenas ${ }^{17}$ y que las únicasbiblio tecasquerealmenteestán en servicio sonlasdePuebla, sepuedeafirmar que sólo el $0.05 \%$ de esas comunidades cuenta con servicios bibliotecarios que se proponenresponderasuspeculiaridades.

\section{PROBLEMAS PARA LA IMPLANT ACIÓN DE SERVICIOSBIBLIOTECARIOS}

Lospro blemasrelativosalaimplantación deserviciosbiblio tecariossemanifiestan enlasco lecciones, en losmismosserviciosyenlosrecursoshumanos. Seconsideraque tan to elperso nal como lasco leccio nessonlospun tosno dales, pero serevisarán en términosgeneraleslosproblemasreferentesalostresaspectosmencionados.

16 María del Ro cío G raniel Parra. LascommidadesindígenasylosserviaosbibliotecaniosenMéxicounestudio decasa Méxica Opat, p. 62-65.

17 Arnulfo Embriz \&al. qu at. 


\section{Colecciones}

Laconformación deco leccionesquefavo rezcaneldiseñoylaoperatividad deser viciosbiblio tecariosadecuados para las comunidades in dígenassehavisto afectada históricamente por la política de castellanización de lo indígena, y ha repercutido también enlafaltadenormalización paralalectoescrituradeal gunaslen guasin dígenasyenlaescasez demateriales.

Sibien existenmaterialesso brelasculturasindígenas, éstoshansido escritospory para expertos estudiosos de lo indígena. En realidad existen pocos materiales para uso de los indígenas, lo que hace que en la conformación deco lec cio nesparalasbi blio tecasindígenasseidentifiquenlossiguientesproblemas: 18

a) Escasa existencia de materiales en las lenguas indígenas y no en el lenguaje adecuado.

b) Sólo haytex tosesco larespara25delaslen guas in dígenas, pero nadaparalasrestantes. Existen también algunas ediciones bilingües de cuentos y leyendas para niños deeducaciónpreescolar. ${ }^{19}$ Esto significaqueno haymaterialesesco laresparaaproximadamente el 60\% de las lenguas restantes, esto es para 39 lenguas indígenas.

c) Son exiguos los materialesso bre los saberes pro pios delascul tu rasydetestimonioshistó ricosqueapo yen aestasco munidades en elreco no cimien to desupropia identidad cultural.

d) Seigno ralaubicación delospo cosmaterialesexisten tesyportan to no haynin gut na difusión de ellos.

\section{Servicios bibbliotecarios}

Lasbiblio tecaspúblicasdestinadasporlaD G B a la comunidades indígenascontem plan tan to losservicios depréstamo (en salayado micilio) como el decon sul ta. Sin embargo en estasco munidadeslosbeneficiosdelosserviciosdepréstamo en sala yado micilio resultanseriamenteafectadosporlafaltadematerialesenlalen gua maternaylafaltadealfabetización entrelosusuarios.

A simismo el servicio deconsultaeinformación oral, in dividualygrupal, den troy fuera de la biblioteca, tan necesario para estas comunidades, no es considerado en esos términos sino que más bien es consideradoteóricamente como un servicio de consulta de corte general para comunidades alfabetas en lengua española. A demás estos servicios están diseñados para prestarse no a grupos comunitarios sino para atenderpreferentementenecesidadesindividuales.

18 Maríadel Ro cío G raniel Parra,.LascommidadesindígenasylosservidosbibliotecariosenMéxicaunestudio decasa Méxica Opait, p. 35 y 233-235.

19 Guz mán Gó mez, Elba,V ocesindígenaseducadónbiling̈̈diailturalenMéxiç México,D D.F., Dirección GeneraldePublicacio nesdelConsejo Nacio nal paralaCulturaylasArtes/ Instituto Nacio nal Indigenista, 1991, $159 \mathrm{p}$. 
En el caso deCESD ERlosserviciosbiblio tecariossonaten didosen cadaco muni dad porperso nalquedo minalalenguamaterna, pero estacaracterísticano bastapara queestosserviciossean eficaces, razón porlacual eseCen tro trabajaen lacapacita cióndesupersonal.

\section{Recursos humanos}

D ebido aquelaDG B hadelegado enlosmunicipioslaresponsabilidad deasignar ypro porcio narel salario al perso nal delasbiblio tecaspúblicas, el perso nal esinadecuadoyacusalassiguientescaracterísticas:

a) Elbiblio tecario queatien delasco munidadesin dígenasno cum plecon elrequisi to de dominar la lengua materna indígena.

b) En elmejordeloscasosel personal tieneunaesco laridadmáximadesecun daria.

c) La mo vilidad del perso nal esmuygran de. Lapermanen ciapro medio esdeaño y medio, situación que relativiza los efectos de la capacitación.

d) El personal bibliotecario en general carece de conocimientos y su actitud fundamen tal no esem páticahacialaaten ción delaco munidadalaquesirve, otro requi sito que es básico.

Aun quelaD G B hacapacitado al perso nalresponsabledelasbiblio tecaspúblicas lo hahecho demaneragenérica, porlo queno hacui dado el do minio delalenguama ternain dígena, ni hacon si derado queesnecesario tenerunbagajeadecuado alascaracterísticas, necesidadesdeinformaciónytiposdeserviciosqueleconvienenaesta población.

En el caso deCE SDER,elpersonalcontratadoreúnelassiguientescaracterísticas:

a) es bilingüe,

b) es oriundo de la misma comunidad que atiende,

c) tiene formación escolar y académica que va desdesecun dariaconcluidahastaes tudiantes de alguna carrera profesional,

d) cuen tacon ciertacapacitación tan to paraestimularlalecturacomo paramanejar básicamente el acervo bibliotecario.

Trascompararlassituacio nesan terio resseponedemanifiesto laurgen tenecesi dad de formar los recursoshumanos calificados para darles servicios bibliotecarios adecuadosalascomunidadesindígenas. 


\section{CARACTERÍSTICAS BÁSICAS DE LOS SE RVICIOS BIBLIOTECARIOS20}

\section{Colecciones}

Alinstrumentarlosserviciosbiblio tecariosparalasco munidadesindígenassede ben in tegrarco lec cio nesquerespon dan, enfon do y forma, asusnecesi dades deinformación.Almismo tiem po estain formación debeestar, obviamen te, enlalen gua quehablalaco munidad.

Elproblemadelaescasaexisten ciadematerialesadecuadoshacemásim perantela búsqueda e identificación de esos materiales para incluirlos en estas colecciones. También es ineludible promover e involucrar al bibliotecario y la comunidad en la pro ducciónyregistro deinformación querequieraso portesfísicosdebajo costo, así como contarconlacolabo ración delasinstitu cionesinvolucradasenlaproblemática indígena.

Losmaterialesdeberánresponderalasnecesidadesdeinformacióndelacomuni dadypro mo verelfortalecimien to delaidentidadin dígenaasícomo preservarein te grarlosco no cimien tosysaberesdeesaco munidad como partedel patrimo nio cultur ral del país.

Estascoleccionestendránqueestartécnicamenteorganizadasparapoderofrecer adecuadamentelosserviciosbibliotecarios.

\section{Servicios bibliotecarios}

Frenteaco munidadesin dígenasconlascaracterísticasylasnecesidadesdeinformaciónarribamencionadas,losserviciosbiblio tecariosquedeberánproporcionarse tendránnecesariamentelassiguientespeculiaridadessiesquehandeserefectivos:

* Elservicio deconsultaeinformación debeprivilegiarlaoralidadylacolecti vidad den tro yfueradelabiblio teca, en los espacios pro piosdelos diferentesgru posco munitarios. Elanal fabetismo realy funcio nal queprivaen esas comunidadesconvierten en requisito queelbiblio tecario, o un tercero alfa betizador, traduzca los materiales escritos a la lengua que habla el usuario.

* Los servicios de préstamo en sala y a domicilio deben permanecer porque benefician a la escasa población de lectoescritores en español.

20 Esteapartado seapoyó sustan cialmenteenlassiguientesregulacionesinternacio nales: 1) Acta Final del Primer Congreso Indigenista Interamericano (celebrado en Pátzcuaro, México, Abril de 1940), 2) Convenio No.169so brepueblosin dígenasytribalesen paísesin depen dien tes(Organi zaciónInternacio naldelTrabajo,1989),3)Convenio No.107so brepo blacio nesin dígenasytriba les (OIT, Ginebra, Suiza, 26 de junio de 1957), 4) Manifiesto de la UNESCO sobre la Biblioteca Pública, 1994(UNESCO , IFLA, 1994), 5)Co munidadesmulticulturales:NormasparaServiciosBi bliotecarios (Federación Internacional de Asociaciones de Bibliotecarios y Bibliotecas, (IFLA), Sección de Servicios Bibliotecarios a Poblaciones Multiculturales, 1989), y 6) Encuentro latinoamericano so brelaA ten ciónBiblio tecariaalasCo munidadesIn dígenas(Encuen trodel 15-17 de noviembre de 2000 en la Ciudad de México). 
* Los servicios bibliotecarios deberán contar, para ser efectivos, con la aceptación de la comunidad mediante la participación de colaboradores.

* Lasnecesidadesdeinformaciónparticularesdelosgruposdeestasco muni dades exigen que tras identificarlos, el bibliotecario las dinamice y priorice continuamente, y que vaya y lleve la información adecuada al encuentro de los usuarios en sus propios espacios grupales.

* La existencia de un órgano rector y coordinador resulta indispensable en cadainstitu ción, gubernamen tal o no, paraquediseñelosserviciosbiblio te carios, lleveaefec to laselección, ad quisiciónypro cesamien to técnico delos materiales, y brinde también asesoría constante a la biblioteca local a la vez que se nutre de la evaluación de ésta frente a la comunidad.

\section{Recursos humanos}

Tantolaprestación deestosserviciosbiblio tecarioscomolaintervención delabi blio tecaenlasal vaguardadelacul turain dígenamarcarán un parteaguassiefec tivamen tesedalaformación debiblio tecariosqueaten derán alasco munidadesin díge nasporseréstaunamo dalidadinterdisciplinaria.

Las características especiales de las comunidades indígenas señaladas en apartados anteriores fundamentan la exigencia de que la información esté codificada en lenguaje sencillo, preferentemente en soportesfísicos de oralidad secundaria en las len guasmaternayespañol,yqueel biblio tecario además deserbilin güeen lalen gua materna de la comunidad que atienda, proporcione la información no sólo escrita sino principal men tedemaneraoralygrupal, lo queim plicaríaque, so bretodo tratándo sedeanalfabetos,éllesleyerapreferentementeengrupo, o seauxiliaraparaello de algúnotromiem brodelacomunidad.

Toda vez que el flujoylaconstrucción delain formación en estasco munidadeses eminentementeco lectivo,oralypresentaunaaltapo tencialidad paracaptarelmen saje co dificado enimágenes, permeado todo ello porladiferenciaciónlingüísticaycultural decadaunadelasetnias, elbiblio tecario deestasco munidadesdeberáconsiderarestrategiasdecomunicacióndeinformacióncomunitariasyresponderensuoperatividad a op cio nesquecon tem plen elpuen tedefortalecimien to delo oralylo escrito en ambas lenguas, elespañolylamaterna.Portanto, seproponeelsiguienteperfil:

* El bibliotecario deberáestar preparado paraidentificarlosmaterialesperti nen tesquecon formarán lasco lec cio nes, pro mo verlaelabo ración demate riales y organizarlos adecuadamente e identificar las necesidades de la comunidad.

* D eberá estar medularmente preparado para operar los servicios y proporcio narlainformación pertinen te, tan to en españolcomo enlalen guamaterna de la comunidad, así como para propiciar la participación de la comunidad en la satisfacción de sus necesi dadesyen ladifusión delainformación propia. 
Esto plantealanecesidad deformarrecursoshumanostanto pro fesio nalescomo técnicos. Elproyec to queaquíestamospresen tan do remitealaformacióndepersonaltécnico.

\section{Propuest A DE FORMACIÓNTÉCNICA}

\section{F undamentación}

Ante la necesidad de una atención bibliotecaria que tome en cuenta la especificidad cul turalylingǘsticadelapo blaciónin dígenamexicana, seconsiderapertinente la formación derecursosbiblio tecariosquerespondanalasconductasinformativas delosindígenas.

Actualmenteno existeperso nalprofesionalbiblio tecario debidamentepreparado quepuedaofrecerleserviciosbibliotecariosadecuadosalasco munidadesin dígenas. Laurgen ciadepreparar, al menostéc nicamen teal perso nal existen teyal quepo tencialmente se abocará a la atención inmediata de tales servicios para esas comunidades, nosllevó aoptarporeldiseño delapresentepro puesta.

Estacapacitaciónseríaim partidaen un curso demo dalidad presenaialcon lain tención defortalecerelapren dizajein dividual apartirdel grupo ein cluiríaal con ductor deésteparafavo recerlariquezadeex perien ciasyenfo ques.Lacapacitaciónim plicaríacuatro módulosqueincluiríanun to talde240horas.

Laprácticabiblio tecariaseríareplan teadaalaluz deun cuerpo deco no cimien tos quetienecomo ejeprincipalladisciplinabiblio teco ló gicapero queseapo yaen aportesdelaantropología,lalingüística,laco municaciónylaso ciología.

Losmó dulosdeberían prestaraten ción conlamayorintegralidad po siblealoscono cimientos, habilidadesyactitudesqueserequiereny, conservarelrigoracadémico y metodológico que favorezca la adquisición de conocimientos de manera lógica y secuenciada.

La noción de integralidad se propone dar respuesta al planteamiento que señala quelaeducación ac tual carecedeunasólidaen señanzaso cialyhumanística ${ }^{21} \mathrm{y}$, por otro lado subrayar la orientación de educación libertadora de Paulo Freire,22 en el sen ti do dequelaedu cación no puedesersólo un depó sito decon tenidossino laproblematización de los asuntos humanos y de sus relaciones con el mundo. Así este proceso deenseñanza-aprendizajepretendedesarrollar la capacidad de problematizarel con tex to deinformación in dígenarural buscan do lasso lu cio nespo siblesme dianteelmanejo deco no cimien tos, habilidadesyactitudes. Tam biénbuscaríaaproximarseal ideal edu cativo plan teado poralfon so Siliceo deformar "al hombreútily deservicio alaco mu ni dad fortalecien do el sen ti do desulibertad, asícomo el desus

21 Alfonso Siliceo. Capadtacónydesamdlodd pessnal. México, D.F., Li mu sa, 1983, p. 15-22.

22 MiguelEsco barG uerrero.PauloFreireylaeducadónlibertadbra México D .F.E dicio nesElCaballito BibliotecaPedagógica SEP, 1985 p. 24-25. 
obligaciones, derechos y respon sabilidades"; puesdeestaforma, con su hacer, ayut daríaalaliberacióndeotros.

Laeducación reto maríaasílosvalo resso cialesquelepermitenalserhumano darle significado asu trabajo ycon tri buirasu desarro llo in tegral, parael cuallas ap titudes sontanimportantescomolasactitudes.

Estaeducaciónno esajenaalasformasdeorganizaciónco munitariaquepractican lasco munidadesin dígenasyqueleshan sido fundamentalespararesolversusnecesi dadesdeinformaciónysuproblemáticadeso brevivencia.

Lapro puestaquedapuesintegradaporelperfilideal del biblio tecarioyaseñalado, el enfoque de una educación problematizadora, la escolaridad de los aspirantes, el dominio oral de la lengua indígena de la comunidad y también por la necesidad de formaragentesquecontribuyanalcambio enlaconductainformativa.

\section{O bjetivo general}

Seesperaqueelparticipan teen estapro puestadeformacióndesarrolleprincipal menteloscono cimientos, habilidadesyactitudesquelepermitan querarlosservicosbibidtecanios Portan to deberáestarpreparado paraiden tificarlosmaterialespertinentes que conformarán las colecciones, promover la elaboración de otros materiales, organizar técnicamente las colecciones de la biblioteca e identificar las necesidades delacomunidad.Tendríaqueestarpreparado nuclearmen teparapro porcionarlainformación pertinenteenformatan to in dividual como grupalyprincipalmenteoral, so bretodo en lafase tran si to riadelaco munidad delo oral alo escrito (en españoly en len guamaterna),ydeberápro piciarlaadecuadaparticipación delaco munidad enla satisfaccióndesusnecesidadesyenladifusióndelainformaciónpropia.

\section{Módulos}

Paralo grarel objetivo anteriorlapro puestadeformación quedaríaintegradapor lossiguientescuatromódulos:

* MÓ DULO I. Comunidades indígenas y sus necesidades de información.

* MÓ D ULO II. Servicios bibliotecarios para las comunidades indígenas.

* MÓDULO III. D esarrollo y organizaciones de las colecciones.

- MÓDULO IV. Servicios de información para las comunidades indígenas.

Laimparticiónyevaluacióndecadamódulollevaríasesentahoras.

\section{O bjetivos y contenidos de los módulos}

\section{Módulo I. Comunidades indígenas y necesidades de información.}

\section{Objeilogeneral}

Intro duciralosparticipan tesen elreco no cimien to delapro blemáticaylascaracterísticasdelasco munidadesin dígenas, asícomo en susnecesidadesdeinformación 
y en familiarizarlosconlastécnicaspararecabaryregistrarlainformaciónpro pia de lacomunidad.

\section{Objeivos espeáficos}

* Identificarlascaracterísticasdelasco munidadesin dígenasfrentealresto de la población nacional.

- Analizar la importancia que tienen las comunidades indígenas dentro de la realidad nacional y el derrotero histórico en el que se han desenvuelto.

* Revalorar el papel que tiene la información en las comunidades indígenas e intentar construir relaciones más simétricas con la sociedad mayoritaria.

* Reco no cerlaim portan ciaquerepresen tael perfildelosusuariosen el dise ño y operatividad de los servicios bibliotecarios.

- O frecerle a los participantes conocimientos básicos para recabar la informaConteridotemático ción pro du cidaporlasco munidadesyregistraréstaen so portesdebajo costo.

1.- Información y democracia en el contexto indígena.

1.1. D errotero y situación de los indígenas en México.

1.2. Marco legal y político indígena.

a. Problemática lingüística indígena.

i. Normalización de lenguas con sus variantes.

ii. D erechos lingüísticos y a la oralidad.

2.- Necesidades de información de las comunidades indígenas.

3.- Recursos de información para las comunidades indígenas.

4.-Institucionesvinculadas a la ob ten ción deinformación paralasco munidadesindígenas.

5.- Méto dosdevalo raciónysistematización delainformación (escritau oral) característica de las comunidades indígenas.

6.- Soportes físicos de bajo costo para el registro de la información.

6.1. Medios sonoros.

6.2. Medios audiovisuales.

6.3. Medios gráficos y escritos.

\section{MóduLo II. Servicios bibliotecarios para comunidades indígenas. Objeivogeneral}

Proporcionarle a los participantes los elementos bibliotecarios para operar los serviciosbiblio tecariosyparareco no ceraéstoscomolosinstrumentosporex celenciaparademo cratizarelac ceso yeluso delainformación porpartedelasco munida desindígenas.Informarsobrelascaracterísticasfundamentalesdedichosservicios.

\section{Objeivos espeéficos}

* Identificar el papel que juega la biblioteca en los procesos de democratización implicados en la información y la lectura.

* Identificar las características generales en cuanto a estructura y funcionamiento, de una biblioteca orientada hacia las comunidades indígenas. 
Ubicar el papel del bibliotecario en la operación de este tipo de servicios. Aplicar las técnicas y procedimientos que precisa la operación de los servicios bibliotecarios para una comunidad indígena.

Analizar la interacción de la comunidad con las otras instituciones involucradas en la problemática indígena buscando la consolidación de los servicios bibliotecarios.

\section{Conteridotemático}

1.-Responsabilidaddelasbiblio tecasparaayudarenelfortalecimien to demo crático.

1.1. Papel que desempeñan las bibliotecas en la democratización de la información para las comunidades indígenas.

1.2. Fortalecimiento de la cultura oral y adopción de la cultura escrita.

1.3. El bibliotecario como promotor del acceso democrático a la información.

2.- Políticas bibliotecarias internacionales para las comunidades indígenas.

2.1. Bibliotecas para comunidades indígenas y bibliotecas públicas.

2.2. Normas para los servicios bibliotecarios de las comunidades indígenas.

3.- Servicios bibliotecarios.

3.1. Préstamo en sala y a domicilio.

3.2. Consulta e información.

3.3. Otrosserviciosemanadosdelavin culación delabiblio tecaconlosproyectos y programas de desarrollo de la comunidad.

3.3.1. Proyectos para transitar de lo oral a lo escrito.

3.3.2. Alfabetización en lenguas franca y materna.

3.3.3. Proyectos para asegurar el flujo de información que va de la sociedad mayoritaria hacia la comunidad indígena y viceversa.

3.3.4. Proyectos para asegurar el flujo de la información interna de los saberes, conocimientos y testimonios propios de esa cultura indígena.

3.4. Promoción y difusión de los servicios.

3.4.1. O rientación al usuario en el uso y la participación en los servicios bibliotecarios.

3.4.1.1. Reglamento de los servicios de la biblioteca.

3.4.1.2. Uso de la biblioteca.

3.4.2. Estrategias de promoción.

3.4.3. Estrategias de difusión.

3.4.4. Evaluación de la difusión y la promoción.

4.- Escenarios de colaboración informativa indígena interinstitucional.

4.1. Identificación de instituciones.

4.2. O btención de financiamiento.

4.3. Coordinación de proyectos y programas. 


\section{Módulo III. Desarrollo y organización de las colecciones. \\ Objeivogeneal}

Pro piciaren losparticipan teslaapro piación deco no cimien tosyhabilidadesque les permitan desarrollar y organizar las colecciones a través de la selección, adquisición,catalo gación,clasificaciónymantenimiento delacervodelabibliotecalo cal.

\section{Objeivos espeéficos}

* Co no cerlosdiferen testiposyformatosderecursosdo cumentalesquepue den conformar el acervo de una biblioteca orientada hacia una comunidad indígena.

* Identificar la importancia de las necesidades de información como soporte fundamental de la selección y adquisición de materiales.

* Apoyarlaad quisición derecursosdo cumen talesatravésdecompra,canjey donación.

* Identificar los elementos que integran un registro catalográfico.

* Efectuarelpro ceso físico delosdistintostiposderecursosdo cumentales.

* Ordenaryman tener losmateriales do cu men tales en el lu garquelesco rres ponde con base en un sistema de clasificación.

* D arlemantenimien to aloscatálo gosmanualesyauto matizadosdelosdife rentes recursos documentales para localizar fácilmente la información.

* Realizar un levantamiento de inventarios de los diferentes tipos de colecciones.

* Mantener el registro estadístico de uso de los servicios y las colecciones como herramientas de evaluación periódica de la biblioteca.

Conteridb tenático

1.- Tipos de colecciones:

1.1. O bras generales.

1.2. O bras de consulta.

1.3. Publicaciones periódicas.

1.4. Materiales no publicados (literatura gris, información oral, información electrónica).

1.5. Materiales audiovisuales.

1.6. Materiales tridimensionales.

2.- Desarrollo de colecciones.

2.1. Necesidades de información y selección.

2.2. Compra, canje y donación.

2.3. Procedimientos de adquisición.

2.3.1. Rutinas de adquisición.

2.3.2. Formatos de pedido manual y automatizado.

2.3.3. Catálogos de adquisición, manuales y automatizados.

3.- Registros catalográficos.

3.1. O bjetivos y funciones. 
3.2. Elementos de los registros.

4.- Clasificación.

4.1. O bjetivo y función.

4.2. E structura general de los sistemas de clasificación.

4.3. O rdenamiento físico de los acervos.

5.- Preparación física de los distintos recursos documentales.

6.- Mantenimiento de catálogos.

6.1. Importancia y tipos de catálogos.

6.2. Reglas de alfabetización de catálogos.

7.- Inventarios y estadísticas.

7.1.0 bjetivos y funciones.

7.2. Rutinas para realizar inventarios.

7.3. Rutinas para el manejo de los registros estadísticos.

MóduLo IV. Servicios de información para las comunidades indígenas Oljeitogeneral

Proporcionarlealosalumnoslosconocimientosyhabilidadesparaprestarlosserviciosdein formaciónquerespondan alasnecesidadesdeinformación delasco mut nidadesindígenas.

Objeivosespeéficos

* Valorar la importancia de la interacción con los usuarios para precisar sus necesidades de información.

* Manejarlasdiversasfuentesdeinformaciónpararesponderoportunamente a las necesidades de los usuarios indígenas.

* Identificarlasfuentesquecon tengan lain formación queelusuario requiere y localizar la más pertinente.

* Proporcionarlelainformaciónal usuario enlostérminosen quelarequiera.

Conteridos temáticos

1.- Importancia de la interacción con los usuarios.

1.1. O bservación del entorno comunitario.

1.1. Entrevista para identificar las necesidades de información del usuario.

2.- Tipos y características de recursos de información.

2.1. D iccionarios y enciclopedias.

2.2. Fuentes de información geográfica.

2.3. Fuentes de información estadística.

2.4. Fuentes de información biográfica.

2.5. Buscadores en Internet.

2.6. Bases de datos y catálogos.

2.7. Recursos particulares para comunidades indígenas.

3.- Estrategias de búsqueda de información.

3.1. Medios manuales.

3.2. Medios automatizados. 
4.- Comunicación de la información al usuario.

4.1. O ral o escrita, en lengua franca o materna, en el vocabulario adecuado.

4.2. Grupal 0 individual.

4.3. Intramuros o extramuros.

\section{Evaluación}

Para evaluar cada módulo se diseñarán y aplicarán los procedimientos y herramientas que permitan iden tificarsi hubo apro piación, porpartedelos alum nos, de los conocimientos, habilidades y actitudes que importan. Además al concluirto dos losmó dulos del curso habráunasesión conjun taen treel grupo dealum nosyelcuerpo de docentes con la intención de recuperar las opiniones frente a los objetivos, con tenidosymeto do lo gíaem pleadaen cadamó du lo asícomo losdel curso en su totalidad,conmirasamejorarelprocedimientogeneral.

\section{C uerpo docente}

Pesealanaturalezadelprogramaeminentementebibliotecológico,elenfoqueque serequiereesin terdisciplinario.Portan to en el primermó dulo seríain dispensable la participación de especialistas de otras disciplinas además de bibliotecólogos. Cada móduloprecisadelsiguientecuerpodocente:

* MÓDULO I: Un bibliotecólogo, un antropólogo,un lingüista y un comunicólogo.

* MÓD ULO II:D osbiblio tecólo gos, uno especialistaenserviciosbiblio teca riosyotro en pro mo ción, difusiónyescenariosdeco labo racióninterinstitur cional indígena.

* MÓDULO III: D os bibliotecólogos, un especialista en desarrollo de colecciones y otro en organización de información.

* MÓDULO IV: Dos bibliotecólogos, un especialista en consulta y otro en uso de información electrónica.

\section{Requisitos de ingreso}

Losaspirantesdeberán cubrirlascaracterísticasqueacontinuaciónsemencio nan:

a) Experiencia en atención bibliotecaria para comunidades indígenas o que estén por ejercer dicha práctica.

b) Escolaridad mínima de preparatoria concluida o experiencia equivalente.

c) D ominio oral de la(s) lengua(s) indígena(s) de la comunidad que atiende 0 atenderá.

d) Manejo delalectoescrituraenla(s)lengua(s)materna(s)co munitaria(s) queatiende 0 atenderá.

Losaspirantesseseleccio naránmedianteun pro ceso llevado acabo porlaco ordi nacióndelprograma. 


\section{Perfil de egreso}

Alterminarlaformacióndelpresenteprogramaelalumnodeberásercapazde:

1) Ubicar la problemática indígena del país y sus servicios bibliotecarios desde una perspectiva interdisciplinaria que interprete su propio contexto.

2)Fun gircomo pro mo tordeluso delainformación en lasco munidadesin dígenas.

3)A po yarel desarro llo deacervosyserviciosbiblio tecariosacordesconlasco muni dades que sirve.

4) Mantener actualizados los catálogos y organizados los acervos de la biblioteca.

5) Proporcionarle a las comunidades los servicios bibliotecarios y de información adecuados.

6)Pro mover, difun diryco labo rar, demanerain terinstitu cio nal, enlacreaciónyfuncionamiento de programas relacionados con el uso, la compilación y el registro delainformaciónen lalen guamaternain dígenain vo lu cradayen castellano, para facilitar la transición de lo oral a lo escrito.

7) Fo men taren trelosmiem bros delaco munidad, laiden tificación, valo ración, sis tematización, registro, difusión y promoción de la información procedentes de ellos y su comunidad.

8)Brin darlein formaciónalosdiferen tesin dividuosygruposdelaco munidad in dí genademaneraoportunayprecisaparaapo yarlarealización desusac tividadesy tareas.

\section{Requisitos de egreso}

ParaobtenerlaconstanciadeconclusióndelpresenteprogramadeFormaciónde biblio tecariosparalasco munidadesindígenas,elparticipantedeberácubrirto dos los mó du los con un mínimo deasisten ciadel $80 \%$, en cadauno deellos, y haberapro ba dolasevaluacionesrequeridasenellos.

\section{A MANERA DE CONCLUSIONES}

Como partedeloscom pro misosdeestainvestigación co lec tivasepreviólaeva luacióndeesteprogramatécnico pormediodeun pilo teo.

La zona nahuat de la SierraN or tedePueblaseríalasededeestepro gramapilo to envirtud dequeen esazonaseen cuen traunadelasinstitu cio nesquehan co labo rado en el presen teproyecto, dequecuen tacon biblio tecasin dígenasquepo sibilitarán la realizacióndelasprácticasdo cen tesydequetienelainfraestructuraparahospedar a losespecialistasqueim partirían estecurso, asícomo alosalum nos deotras co mu ni dadesqueaspirenalo mismo.

Conelfin deafinarso bretodo lapertinen ciadeloscon tenidosylameto do lo gíay suadecuaciónabiblio tecasybiblio tecariosdeco munidadesin dígenasmexicanas, se esperaqueen estecurso seformen tan to biblio tecariosparalazonadelaSierraNorte dePueblacomoparaotrascomunidadesindígenasdelpaís. 
Enestesentido sepreten dequelosbeneficiosesperadosparaesteproyecto im pli carían afinarlo yformarun primergrupo debiblio tecariostéc nicosparaco munida desindígenasenelpaís.

\section{BIBLIOGRAFÍA}

"Acta final del primer congreso indigenista interamericano" (celebrado en Pátzcuaro, Méxi co, A bril de 1940). En Suplementodd BdeénIndigenis ta.México:Instituto IndigenistaInteramericano,marzo 1948,p.18-19.

Barry J. Smith y Brian, L. El abc dela capadtaión prádica México D .F., MCGRAW-HILL, 1990.

Bonfil Batalla, Guillermo. Méxiop prounda una đivilizacónnneegda. México, Editorial G rijalvo, 1989, 250 p.

BonfilBatalla,G uillermo.Pensarnuestraailtura. México;Ensayos, Alianza Editorial, Col. Estudios, 1991, 172 p.

"Comunidades multiculturales: normas para servicios bibliotecarios" (Federación Internacional de Asociaciones de Bibliotecarios y Bibliotecas (IFLA), Sección de Servicios Bibliotecarios a Poblaciones Multiculturales,1989). En EducadónyBiblicteca, No viem bre 1994, Año 6, No. 51 (extraordinario), p. 43-48.

"Convenio no. 169so brepueblosin dígenasytribales en paísesin dependientes" (O rganización Internacional del Trabajo, 1989). En Améica Indígma, Julio-D iciembre 1996, Vol. 58, No. 3-4, p. 37.

"Convenio no. 107 sobre poblaciones indígenas y tribales" (O IT, Ginebra, Sui za, 26 deju nio de 1957). EnInformaáónsobreDerechosHumanos, p. 1. Sitio de web:

http:// www.ser.gob.mx/ derechoshumanos/ CV107.htm.

DelPozo D el gado,Pilar. Formadóndeformadores Madrid-España,Pirámi de, 1987.

Enarentrolatimoamericanosobrelaatenaónbibliotecariaalascommicadesindígenas (Encuentro del 15-17 de noviembre de 2000 en la Ciudad de México). Compilado por G raniel Parra, María del Rocío. México, D.F., UNAM, Centro UniversitariodeInvestigaciones Bibliotecológicas,Federación Internacional de Asociaciones de Bibliotecarios e Instituciones, 2001. p. 180-182. Memoria.

Em briz,A rnul fo,Arce,Luis etal.Indicadbressociceconómicosdelospuedlosindł́ grasdeMéxico, México,D .F.Institu to Nacio nalIn digenista, D irección de investigación y promoción cultural, Subdirección de investigación, 1993, $246 \mathrm{p}$. 
Esco barG uerrero, Miguel.PauloFrerreylaEducacónLibetadaa. (Biblio te ca Pedagógica SEP), México D.F., El Caballito, 1985, p. 24-25.

Granados A., Jaime. Capaataióny desamdlodd pesanal. México D .F., Trillas, 1999.

G raniel Parra, Maríadel Ro cío. "Aspec toslin güísticosacon sideraren bi blio tecasdirigidasalascomunidadesin dígenasmexicanas". En Mádobs deInfamadón 6 (32-33), septiembre 1999, p. 51-67.

G raniel Parra, Maríadel Ro cío.Lascommidadesindígenasylosserviciosbibliote cariosenMéxicaunestudiodbcasa México:LaA u to ra, 2002. (Tesis- Maestraen Biblio teco lo gía- UNAM, Facultad deFilo so fíayLetras, Maestría en Biblioteclogía). $367 \mathrm{p}$.

G uz mán G o mez, Elba. V ocesindígenaseducadónbiling̈̈dialturalenMéxica México: Dirección General de Publicaciones del Consejo Nacional paralaCulturaylasArtes, Instituto Nacio nal Indigenista,1991,159p.

Korsbaek, Leif. Introduciónal sistema decargos antoloǵa Toluca: Universidad Autónoma del Estado de México, 1996, 316 p.

Ló pezA ustin,Alfredo Cuepohumanoeideologá:lasconcepcionesdlosantiguos nahuas 3er. Ed., Tomo I. México: UNAM, Instituto de Investigaciones Antro po ló gicas,1989.489p.Etnología/ Historia,SerieAntro pológica, 39.

Manifiesto dela UNESCO sdbrela biblidteca púdica 1994 (UNESCO, IFLA, 1994).Hojaplegada. Traduc ción de:Fun dación G ermán Sán chez Rui pérez.

Molina, Alicia. Diálogpeinteración en e procesopedagógico México D.F., El Caballito Biblioteca Pedagógica SEP, 1985.

PolíticasingüísticasenMéxicoCom pilado porG arzaCuarón, Beatriz. Méxi Co: UNAM, Centro de Investigaciones interdisciplinarias en Ciencias y Humanidades, 1997, 270 p. Colección: La democracia en México.

Siliceo,Alfonso. CapaatadaónyDesamdloddPersanal.México D .F.,Limusa, 1983, p. 15-22.

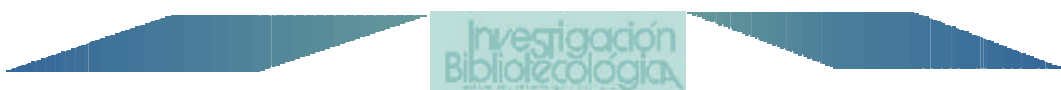

